\title{
Antipruritic Effect of Natural Coumarin Osthole through Selective Inhibition of Thermosensitive TRPV3 Channel in the Skin
}

\author{
Xiao-Ying Sun, Li-Lan Sun, Hang Qi, Qin Gao, Gong-Xin Wang, Ning-Ning Wei, \\ and KeWei Wang
}

Department of Pharmacology, Qingdao University School of Pharmacy, Qingdao, China

Received March 8, 2018; accepted July 16, 2018

\begin{abstract}
Coumarin osthole is a dominant bioactive ingredient of the natural Cnidium monnieri plant commonly used for traditional Chinese herbal medicines for therapies and treatments including antipruritus and antidermatitis. However, the molecular mechanism underlying the action of osthole remains unclear. In this study, we report that osthole exerts an antipruritic effect through selective inhibition of $\mathrm{Ca}^{2+}$-permeable and thermosensitive transient receptor potential vanilloid 3 (TRPV3) cation channels that are primarily expressed in the keratinocytes of the skin. Coumarin osthole was identified as an inhibitor of TRPV3 channels transiently expressed in HEK293 cells in a calcium fluorescent assay. Inhibition of the TRPV3 current by osthole and
\end{abstract}

its selectivity were further confirmed by whole-cell patch clamp recordings of TRPV3-expressing HEK293 cells and mouse primary cultured keratinocytes. Behavioral evaluation demonstrated that inhibition of TRPV3 by osthole or silencing by knockout of the TRPV3 gene significantly reduced the scratching induced by either acetone-ether-water or histamine in localized rostral neck skin in mice. Taken together, our findings provide a molecular basis for use of natural coumarin osthole from the C. monnieri plant in antipruritic or skin care therapy, thus establishing a significant role of the TRPV3 channel in chronic itch signaling or acute histaminedependent itch sensation.

\section{Introduction}

Itch or pruritus is an unpleasant cutaneous sensation that evokes the urgent desire to scratch. Itching can intensify when scratching aggravates lesions of the skin (Ikoma et al., 2006). Although acute itch is considered to be a defense mechanism that alerts the body to remove irritants, transient itching can progress to become persistent and chronic (Zhang, 2015). Chronic itch can be a widespread symptom of systemic diseases, including atopic dermatitis, liver disease, kidney failure, cholestasis, diabetes, and cancers (Mollanazar et al., 2016; Kittaka and Tominaga, 2017). Due to its complex underlying mechanism, chronic itch as an unmet medical need presents a serious health issue which affects upward of $20 \%$ of people worldwide (Meng and Steinhoff, 2016).

Previous studies have shown that the nonselective calciumpermeable transient receptor potential vanilloid 1 (TRPV1), transient receptor potential vanilloid 4 (TRPV4), and transient receptor potential ankyrin 1 (TRPA1) channels play important roles in the transduction of itch sensation (Shim et al., 2007; Wilson et al., 2011; Lucaciu and Connell, 2013;

This work was supported by grants awarded to K.W.W. from the Natural Science Foundation of China [81573410] and the Ministry of Science and Technology of China [2014ZX09507003-006-004] and to X.-Y.S. from the Qingdao Postdoctoral Application Research Project [2015154], and by the Natural Science Foundation of Shandong Province [ZR2017BH020].

https://doi.org/10.1124/mol.118.112466.
Chen et al., 2016; Sun and Dong, 2016). Our and others' identification of gain-of-function mutations in the human transient receptor potential vanilloid 3 (TRPV3) channel from Olmsted syndrome patients with severe itching has unveiled a critical role of the TRPV3 channel in itch sensation and signaling (Lai-Cheong et al., 2012; Lin et al., 2012), and silencing the TRPV3 gene in rodent $\mathrm{ICR}^{\mathrm{TRPV} 3-I-}$ mice attenuates chronic dry-skin pruritus (Yamamoto-Kasai et al., 2012). All these observations indicate that selective inhibition of TRPV3 activity may present a novel strategy for antipruritic therapy (Steinhoff and Bíró, 2009; Wang and Wang, 2017).

The Cnidium monnieri plant is widely used in the clinical practice of traditional Chinese medicine as a remedy for ringworm, male impotence, and blood stasis ( $\mathrm{Li}$ et al., 2015). The main ingredients of $C$. monnieri fructus are coumarins, such as osthole, xanthotoxin, and imperatorin (Dien et al., 2012), and the methanol extracts of fruits of C. monnieri show strong antipruritic activity (Basnet et al., 2001) and are used for anti-itch in skin care products (Li et al., 2015). It has been reported that osthole has an antiallergic effect on allergic animal models (Matsuda et al., 2002). However, whether and how coumarin osthole from $C$. monnieri relieves chronic itch remains largely unknown.

In the present study, we sought to investigate the effect of coumarin osthole on pruritus and its underlying mechanism.

ABBREVIATIONS: AEW, acetone-ether-water; 2-APB, 2-aminoethoxydiphenyl borate; DMSO, dimethylsulfoxide; GSK101, GSK1016790A; GSK1016790A, (N-((1S)-1-\{[4-((2S)-2-\{[(2,4-Dichlorophenyl)sulfonyl]amino\}-3-hydroxypropanoyl)-1-piperazinyl]carbonyl\}-3-methylbutyl)-1-benzothiophene2-carboxamide; hTRPV3, human TRPV3; OS, Olmsted syndrome; RR, ruthenium red; TRPA1, transient receptor potential ankyrin 1; TRPV1, transient receptor potential vanilloid 1; TRPV3, transient receptor potential vanilloid 3; TRPV4, transient receptor potential vanilloid 4; VEH, vehicle. 
Our observations show that osthole specifically inhibits the TRPV3 channel and attenuates scratching behavior induced by either acetone-ether-water (AEW) or histamine in mice. Our findings not only provide a molecular explanation for the key ingredient osthole from plant C. monnieri, traditionally used for anti-itch therapy or skin care products, but also reveal a pivotal role of TRPV3 in chronic itch or acute histaminedependent itch sensation.

\section{Materials and Methods}

Animals. C57BL/6 male mice (6-8 weeks old) were purchased from Vital River Laboratory Animal Technology Co., Ltd. (Beijing, China), and all in vivo experiments were carried out at least 7 days after their acclimation to the housing environment. TRPV3-knockout mice were gifts from Dr. Yong Yang (Peking University First Affiliated Hospital, Beijing, China) and Ardem Patapoutian (Scripps Research Institute, La Jolla, CA). TRPV3-knockout mice were genotyped by polymerase chain reaction according as the protocol reported previously (Moqrich et al., 2005). Mice were housed in a room under controlled temperature $\left(22^{\circ} \mathrm{C} \pm 2^{\circ} \mathrm{C}\right)$ and a 12 -hour light-dark cycle. Food and water were freely accessible to mice. All animal tests were authorized by the Institutional Animal Care and Use Committee of Qingdao University Health Science Center and were performed in accordance with institutional and national guidelines for the use and care of animals for experiments.

Compounds. Osthole (molecular weight: 244.29) was obtained from Shanghai Tauto Biotech Co., Ltd. (Shanghai, China). 2-Aminoethoxydiphenyl borate (2-APB), capsaicin, GSK1016790A [GSK101 (N-((1S)-1-\{[4-((2S)-2-\{[(2,4-Dichlorophenyl)sulfonyl]amino\}3-hydroxypropanoyl)-1-piperazinyl]carbonyl\}-3-methylbutyl)-1-benzothiophene-2-carboxamide)], allyl isothiocyanate, and ruthenium red (RR) were purchased from Sigma-Aldrich (St. Louis, MO). The purity of each standard compound was no less than $98 \%$ by high-performance liquid chromatography analysis. With the exception of ruthenium red, which was dissolved in water, osthole $(100 \mathrm{mM}), 2$-APB $(1 \mathrm{M})$, and GSK101 $(500 \mu \mathrm{M})$ were dissolved in dimethylsulfoxide (DMSO), with the final highest concentration of DMSO not exceeding $0.2 \%$. Capsaicin $(100 \mathrm{mM})$ was dissolved in absolute ethanol. For intracellular calcium measurement, all compounds were diluted in Hanks' balanced salt solution. Compounds used in the electrophysiological experiments were diluted in normal perfusion solution. Compounds used in the behavior experiments were diluted in saline.

Cell Culture and Transient Transfection. HEK293 (the human embryonic kidney cell) was cultured in Dulbecco's modified Eagle's medium supplemented with $10 \%$ fetal bovine serum at $37^{\circ} \mathrm{C}$ with $5 \%$ $\mathrm{CO}_{2}$. HEK293 cells were grown in 12-well plates for subsequent intracellular calcium measurement. HEK293 cells were planted on glass coverslips for subsequent whole-cell patch clamp recordings. HEK293 cells were transiently transfected with human TRPV1 cDNA, human TRPV3 (hTRPV3) cDNA, human TRPV4 cDNA, and human TRPA1 cDNA using Lipofectamine 2000 (Invitrogen, Carlsbad, CA). A green fluorescence protein reporter plasmid was cotransfected with an individual TRP channel for patch clamp recordings.

Preparation and Culture of Mouse Keratinocytes. Newborn C57BL/6 mice (P0-P2) were sacrificed and soaked in 10\% povidoneiodine for 5 minutes. After rinsing in $75 \%$ ethanol and phosphatebuffered saline multiple times, the skin was removed and placed in $0.25 \%$ trypsin for $14-16$ hours at $4^{\circ} \mathrm{C}$. Keratinocytes were filtered through a $40-\mu \mathrm{M}$ cell strainer and placed on $2 \%$ gelatin-coated glass coverslips or dishes. Keratinocytes were grown in minimum essential medium containing $10 \%$ fetal bovine serum and $0.002 \%$ primocin at $5 \% \mathrm{CO}_{2}$ and $37^{\circ} \mathrm{C}$ (Cheng et al., 2010; Chen et al., 2016).

Intracellular Calcium Measurement in FlexStation 3 Assay. Variations in intracellular calcium $\left(\left[\mathrm{Ca}^{2+}\right]_{\mathrm{i}}\right.$ ) levels in cells were measured by calcium fluorescent dyes using the Cal-520 PBX Calcium Assay Kit (AAT Bioquest, Sunnyvale, CA) in a FlexStation
3 Multi-Mode Microplate Reader (Molecular Devices, San Jose, CA). HEK293 cells transfected with TRP channel cDNAs were seeded in 96-well black-walled plates (Thermo Fisher Scientific, Waltham, MA) at a density of $\sim 40,000$ cells/well and grown overnight at $37^{\circ} \mathrm{C}$ with $5 \%$ $\mathrm{CO}_{2}$. Cells were loaded with dye supplied with the Cal-520 PBX Calcium Assay Kit for 1.5 hours at $37^{\circ} \mathrm{C}$. Loading and imaging were performed in Hanks' balanced salt solution (137 mM NaCl, $5.4 \mathrm{mM}$ $\mathrm{KCl}, 0.4 \mathrm{mM} \mathrm{KH} \mathrm{PO}_{4}, 0.1 \mathrm{mM} \mathrm{Na} \mathrm{HPO}_{4}, 1.3 \mathrm{mM} \mathrm{CaCl}_{2}, 0.8 \mathrm{mM}$ $\mathrm{MgSO}_{4}, 5.5 \mathrm{mM}$ glucose, $4 \mathrm{mM} \mathrm{NaHCO}_{3}$, and $20 \mathrm{mM}$ HEPES, $\mathrm{pH}$ 7.4). Fluorescent intensity at $525 \mathrm{~nm}$ was measured at an interval of 1.6 seconds using an excitation wavelength at $485 \mathrm{~nm}$ and an emission wavelength at $515 \mathrm{~nm}$ (Lei et al., 2013; Wei et al., 2016).

Whole-Cell Patch Clamp Recordings. Whole-cell recordings were performed using a HEKA EPC10 amplifier with PatchMaster software (HEKA, Instrument Inc, Lambrecht/Pfalz, Germany). The glass pipettes were pulled using a puller (DMZ-Universal, ZeitzInstruments $\mathrm{GmbH}$, Martinsried, Germany) with a resistance of 4-5 $\mathrm{M} \Omega$. For ramp recordings of HEK293 cells or primary keratinocytes, both pipette solution and bath solution contained $130 \mathrm{mM} \mathrm{NaCl}$, $0.2 \mathrm{mM}$ EDTA, and $3 \mathrm{mM}$ HEPES (pH 7.2) (Cao et al., 2012). Membrane potential was held at $0 \mathrm{mV}$, and the current in response to $500-\mathrm{ms}$ voltage ramps was from -100 to $+100 \mathrm{mV}$. For whole-cell patch clamp recordings of the dose-dependent action of osthole on TRPV3 channels, current was elicited by a $400-\mathrm{ms}$ step to $+80 \mathrm{mV}$ followed by a $400-\mathrm{ms}$ step to $-80 \mathrm{mV}$ at 2 -second intervals. Current was analyzed at $\pm 80 \mathrm{mV}$. All recording data were analyzed with Origin 8.6 (OriginLab, Northampton, MA).

Evaluation of Scratching Behavior in Mice. For assessing itch behaviors, the hair of the rostral part of the mouse right neck was shaved using electric hair clippers 24 hours before starting experiments. The behaviors were recorded on video before analysis. The number of bouts of scratching was counted by playing and watching the recorded videos. One bout of scratching was defined as an episode in which a mouse lifted its right hind limb toward the injection site and scratched continuously for any length of time until this limb was returned to the floor or mouth (Wilson et al., 2013). All animal experiments were conducted with laboratory assistants who were blinded to experimental conditions and unfamiliar with the experimental treatment.

For the AEW test, the mouse right neck was treated twice a day with a cotton swab immersed in a 1:1 mixture of acetone and ether or water for 15 seconds, followed by water for 30 seconds for a duration of 5 days (Miyamoto et al., 2002; Yamamoto-Kasai et al., 2012; Wilson et al., 2013). Mice were placed in a box $(9 \times 9 \times 13 \mathrm{~cm})$ for approximately 30 minutes for acclimatization on the morning of day 6 . For osthole treatments, vehicle $(0.0003 \%$ DMSO) and osthole $(3,30,300 \mathrm{nM})$ were injected intradermally into the mouse neck. All compounds were injected in a volume of $50 \mu \mathrm{l}$. Behaviors were recorded on video for 60 minutes following the injection of osthole.

For the histamine test, mice were placed in a box $(9 \times 9 \times 13 \mathrm{~cm})$ for approximately 30 minutes for acclimatization. Saline and histamine $(100 \mu \mathrm{M})$ were injected intradermally into the neck in a volume of $50 \mu \mathrm{l}$. In the inhibited experiments, vehicle (0.0003\% DMSO) and osthole (30, $300 \mathrm{nM}$ ) were injected 30 minutes before the intradermal injection of histamine. Behaviors were recorded on video for $30 \mathrm{~min}$ utes following the injection of histamine (Yang et al., 2016).

For the locomotor activity test, mice were placed in clear open-field chambers equipped with infrared photo sensors after osthole $(3,30$, $300 \mathrm{nM}, 50 \mu \mathrm{l}$ ) was injected intradermally into the mouse neck. Locomotor activity was recorded for 60 minutes of the total distance traveled in the chamber.

Histology. The skin of the mouse right neck was dissected immediately after sacrifice, and skin tissues were then fixed in $4 \%$ paraformaldehyde overnight at $4^{\circ} \mathrm{C}$, dehydrated in ethanol, and embedded in paraffin (Wilson et al., 2013). Sections $(5 \mu \mathrm{m})$ were stained with H\&E. Slides were imaged with a bright-field microscope (ECLIPSE Ti-S; Nikon, Tokyo, Japan) with a charge-coupled device camera (DS-Ri2; Nikon). 
Statistical Analysis. All data are expressed as the means \pm S.D. using GraphPad Prism 5.0 software (GraphPad Software, La Jolla, CA). Statistical significance for two-group comparison was assessed by Student's $t$ test (two-tailed, unpaired). One-way analysis of variance followed by a multiple-comparison test was used to evaluate multiple test treatments. A value of $P<0.05$ was considered to be statistically significant. $\mathrm{IC}_{50}$ is the concentration determined for half-maximal inhibitory effect.

\section{Results}

Inhibition of TRPV3 Channel by Natural Coumarin Osthole in HEK Cells Overexpressing TRPV3. We started investigating the effect of coumarin osthole isolated from the C. monnieri plant on calcium influx induced by activation of hTRPV3 channels expressed in HEK293 cells in a calcium fluorescent assay using the FlexStation 3 Multi-Mode
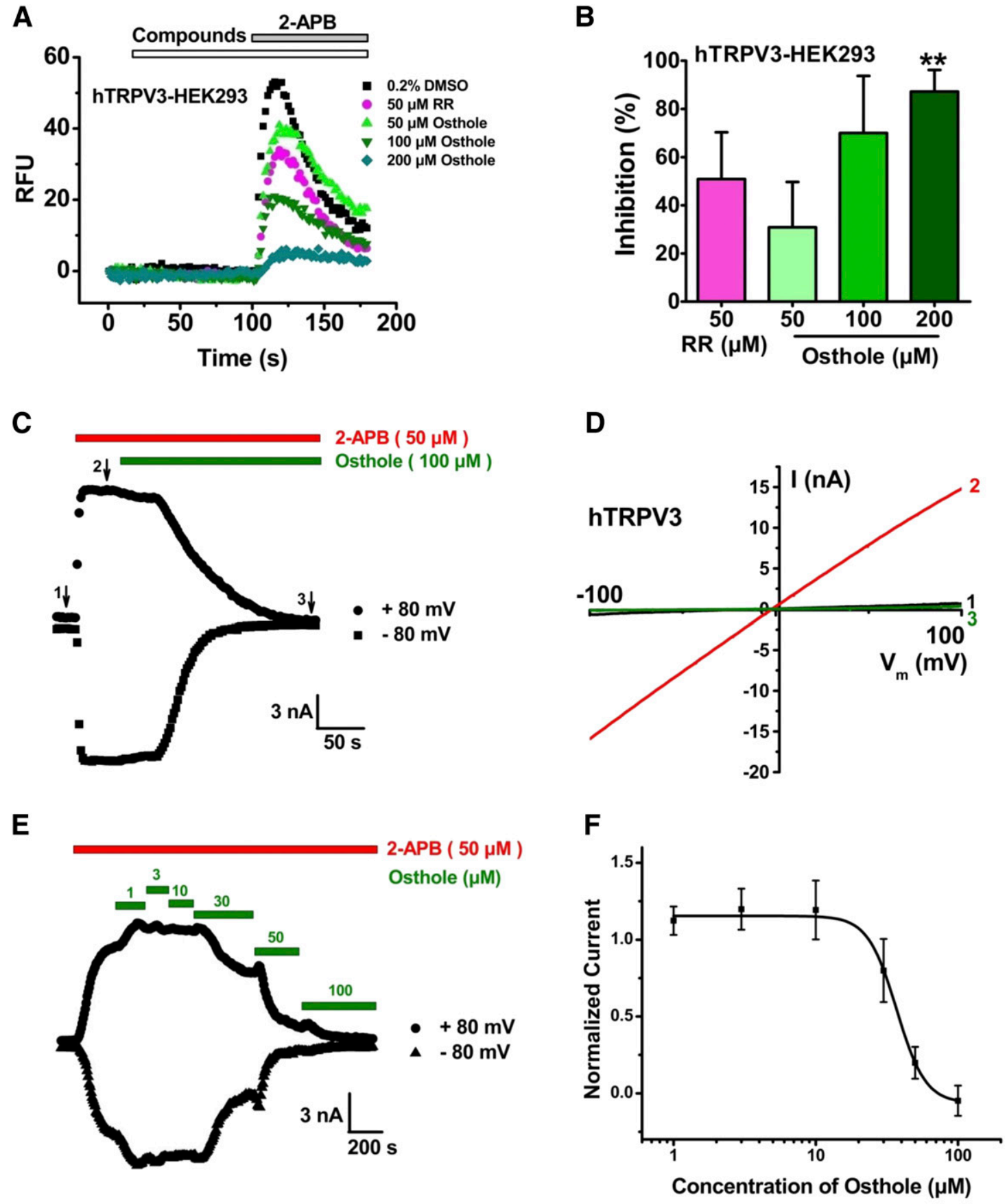

Fig. 1. Osthole inhibits TRPV3-mediated $\mathrm{Ca}^{2+}$ influx and current activated by 2-APB. (A and B) Different concentrations of osthole ranging from 50 to $200 \mu \mathrm{M}$ reduced intracellular $\mathrm{Ca}^{2+}$ increases in response to $200 \mu \mathrm{M}$ 2-APB in hTRPV3-transfected HEK293 cells. Data are expressed as the means \pm S.D Asterisks denote statistical significance compared with RR control $\left(n=6 ;{ }^{* *} P<0.01\right.$ by one-way analysis of variance followed by Dunnett's test). (C) 2-APB ( $50 \mu \mathrm{M}$, red bar) evoked the current increase. The current was completely inhibited by coapplication of $100 \mu \mathrm{M}$ osthole (green bar). (D) Currentvoltage curves of hTRPV3 in response to voltage ramps from -100 to $+100 \mathrm{mV}$ under control conditions (1), 50 $\mu \mathrm{M} \mathrm{2-APB} \mathrm{(2),} \mathrm{and} \mathrm{after} \mathrm{coaddition} \mathrm{of}$ $100 \mu \mathrm{M}$ osthole and $50 \mu \mathrm{M}$ 2-APB (3). (E) Representative hTRPV3 currents in response to $50 \mu \mathrm{M} 2$-APB were inhibited by increasing concentrations of osthole from 1 to $100 \mu \mathrm{M}$. (F) Curve fitting analysis of dose-dependent inhibition of hTRPV3 by osthole with an $\mathrm{IC}_{50}$ of $37.0 \pm 1.9 \mu \mathrm{M}(n=4)$. Data are presented as the means \pm S.D. RFU, relative fluorescence unit. 

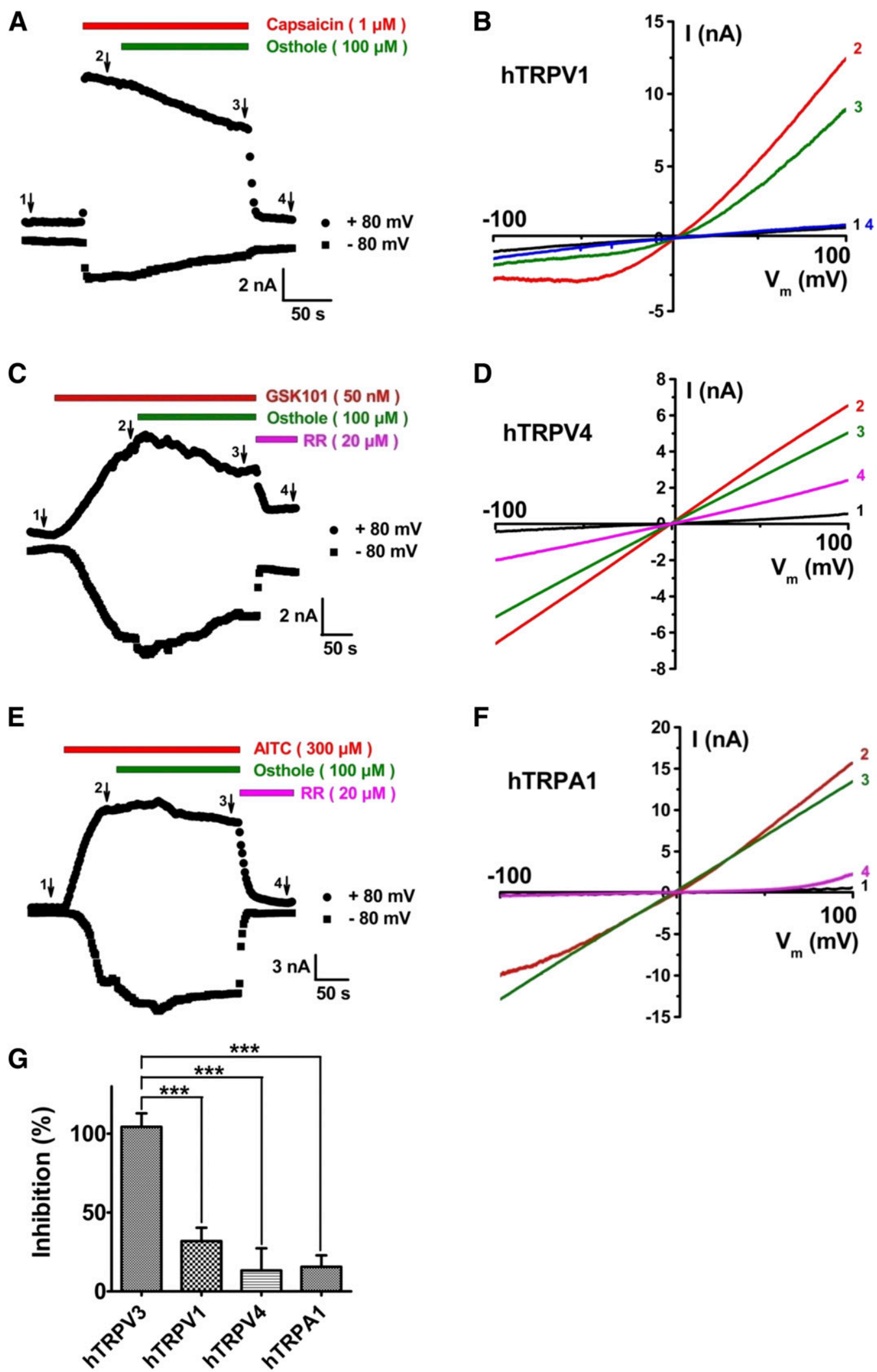

Fig. 2. Selectivity of osthole for TRPV3 over TRPV1, TRPV4, and TRPA1 channels. (A) human TRPV1 current was activated by $1 \mu \mathrm{M}$ capsaicin (red bar). The current was partially inhibited by coapplication of $100 \mu \mathrm{M}$ osthole (green bar). (B) Current-voltage curves of human TRPV1 (hTRPV1) in response to voltage ramps from -100 to $+100 \mathrm{mV}$ under control conditions (1), $1 \mu \mathrm{M}$ capsaicin (2), after coaddition of $100 \mu \mathrm{M}$ osthole and $1 \mu \mathrm{M}$ capsaicin (3), and washout (4). (C) Human TRPV4 (hTRPV4) current was activated by $50 \mathrm{nM}$ GSK101 (red bar) or in the presence of $100 \mu \mathrm{M}$ osthole (green bar) before inhibition by $20 \mu \mathrm{M} R R$ (pink bar). (D) Current-voltage curves of human TRPV4 in response to voltage ramps from -100 to $+100 \mathrm{mV}$ under conditions of control (1), $50 \mathrm{nM}$ GSK101 (2), coaddition of $100 \mu \mathrm{M}$ osthole and $50 \mathrm{nM}$ GSK101 (3), and $20 \mu \mathrm{M}$ RR (4). (E) Human TRPA1 (h TRPA1) current was activated by $300 \mu \mathrm{M}$ allyl isothiocyanate (AITC, red bar) or in the presence of $100 \mu \mathrm{M}$ osthole (green bar) before complete inhibition by $20 \mu \mathrm{M}$ RR (pink bar). (F) Current-voltage curves of human TRPA1 in response to voltage ramps from -100 to $+100 \mathrm{mV}$ under conditions of control (1), $300 \mu \mathrm{M}$ AITC (2), 
Microplate Reader. The compounds were individually added onto cells in a 96 -well plate at 17 seconds before subsequent addition of TRP channel agonist at a time point of 100 seconds, and the relative fluorescence unit values were continuously measured for 80 seconds. As shown in Fig. 1, we identified an osthole that decreased the level of $\mathrm{Ca}^{2+}$ influx induced by TRPV3 agonist 2-APB $(200 \mu \mathrm{M})$ in hTRPV3-transfected HEK293 cells (Fig. 1, A and B). Increasing concentrations of osthole $(50,100,200 \mu \mathrm{M})$ resulted in a dose-dependent inhibition of TRPV3 channels activated by $200 \mu \mathrm{M} 2$-APB $(n=6, P<0.01)$ as compared with the nonspecific TRP channel blocker RR $(50 \mu \mathrm{M})$. To further confirm the inhibitory effect of osthole on TRPV3, we performed the whole-cell patch clamp recordings of hTRPV3-transfected HEK293 cells. Osthole at $100 \mu \mathrm{M}$ completely inhibited TRPV3 current activated by $50 \mu \mathrm{M}$ 2-APB (Fig. 1, C and D). Application of different concentrations of osthole from 1 to $100 \mu \mathrm{M}$ resulted in a dose-dependent inhibition of TRPV3 current induced by $50 \mu \mathrm{M} 2$-APB (Fig. 1E). Fitting analysis revealed that osthole inhibited TRPV3 in a dose-dependent manner with an $\mathrm{IC}_{50}$ value of $37.0 \pm 1.9 \mu \mathrm{M}$ and Hill coefficient of 1.15 (Fig. 1F). These results indicate that coumarin osthole dose-dependently decreased $\mathrm{Ca}^{2+}$ influx and current through activation of TRPV3 induced by agonist 2-APB.

Osthole Selectively Inhibits TRPV3 Current. To evaluate the selectivity of osthole, we tested the effect of osthole on TRPV1, TRPV4, and TRPA1 channels individually expressed in HEK293 cells by whole-cell patch clamp. Currents were elicited by $500-\mathrm{ms}$ voltage ramps from -100 to $+100 \mathrm{mV}$. Osthole at $100 \mu \mathrm{M}$ partially inhibited the TRPV1 current activated by the specific agonist capsaicin $(1 \mu \mathrm{M})$ about $32.0 \%$ $\pm 8.4 \%$ (Fig. 2, A, B, and G) and inhibited the TRPV4 current activated by the specific agonist GSK101 (50 nM) only about $13.3 \% \pm 14.1 \%$ (Fig. 2, C, D, and G). Similarly, osthole at $100 \mu \mathrm{M}$ caused a slight inhibition of the TRPA1 current induced by $300 \mu \mathrm{M}$ allyl isothiocyanate about $15.6 \% \pm 7.2 \%$ (Fig. 2, E-G) as compared with the same concentration of osthole that resulted in complete inhibition of the TRPV3 current $(104.4 \% \pm 8.6 \%, n=5, P<0.001)$ (Fig. 2G). All of these results confirm that natural coumarin osthole is a selective inhibitor of the TRPV3 channel.

Osthole Inhibits TRPV3-Like Current in Mouse Primary Cultured Keratinocytes. TRPV3 is abundantly expressed in primary cultures of keratinocytes (Peier et al., 2002; Xu et al., 2006). To further confirm the inhibitory effect of osthole on endogenous TRPV3, we isolated keratinocytes from the skin of newborn C57BL/6 mice. Application of TRPV3 agonist cocktail $(200 \mu \mathrm{M} 2$-APB $+500 \mu \mathrm{M}$ carvacrol) to primary cultured keratinocytes induced TRPV3-like currents (Fig. 3A), consistent with observations by others (Cheng et al., 2010). RR at $20 \mu \mathrm{M}$ completely inhibited agonist-activated inward TRPV3 current, and reduced the outward TRPV3 current by $65.1 \% \pm 11.4 \%$ (Fig. 3 , $\mathrm{A}, \mathrm{B}$, and E). Osthole at $200 \mu \mathrm{M}$ completely inhibited the inward and outward TRPV3 current induced by $200 \mu \mathrm{M}$ 2 -APB $+500 \mu \mathrm{M}$ carvacrol (Fig. 3, C-E). These results further confirm that natural osthole is an inhibitor of the native TRPV3 channel.

Osthole Attenuates Scratching Behavior Induced by AEW Treatment. A previous study reported that TRPV3 has a significant role in the development of itch in mice with dry skin (Yamamoto-Kasai et al., 2012). To examine the antipruritic effect of osthole, we used a mouse chronic pruritus model of dry skin induced by the mixture of AEW (Miyamoto et al., 2002; Wilson et al., 2013). After an AEW treatment of 5 days, the mouse skin became dry with white dandruff as compared with water vehicle (VEH) control (Fig. 4, A and B). Histologic examination of skin sections confirmed that mice treated with AEW developed an epidermal thickening $(39.3 \pm 1.9 \mu \mathrm{m}, n=$ 3 , for AEW) in the skin isolated from the nape of neck as compared with control (11.4 $\pm 0.6 \mu \mathrm{m}, n=3$, for VEH), a hallmark of psoriatic chronic itch in humans (Fig. 4, C and D). On day 6 , vehicle DMSO $(0.0003 \%)$ and different concentrations of osthole $(3,30,300 \mathrm{nM}$ in $50 \mu$ l per site) were intradermally injected into the mouse neck after 30-minute acclimatization. The scratching behavior was recorded for 60 minutes following the injection of DMSO and osthole. Behavioral evaluations showed that the number of spontaneous scratching bouts in AEW-treated mice significantly increased to $44.3 \pm 19.4$ per 60 minutes $(n=8, P<0.001)$ compared to that of water VEH control at $7.8 \pm 8.0(n=9)$ (Fig. 4E). By contrast, intradermal injection of osthole caused a dose-dependent inhibition of scratching bouts within a period of 60 minutes. Osthole at 30 and $300 \mathrm{nM}$ resulted in a significant reduction of scratching bouts to $27.1 \pm 8.1(n=8$, $P<0.05)$ and $17.3 \pm 8.4(n=10, P<0.001)$, respectively (Fig. $4 \mathrm{E})$.

To test whether osthole could induce a sedative effect, locomotor activity was assessed within a 60-minute session after intradermal injection of osthole into the nape of the mouse neck. Administration of different concentrations of osthole (3, 30, $300 \mathrm{nM}$ in $50 \mu$ l per site) showed no significant difference in total travel distance (Fig. $4 \mathrm{~F}$ ). These results indicate that inhibition of TRPV3 by osthole attenuates dry skin-induced chronic pruritus.

Osthole Suppresses Histamine-Induced Scratching. As the most common pruritogen, histamine can produce itch associated with wheal and flare in human skin (Sun and Dong, 2016). Yang et al. (2016) recently reported that osthole inhibited histamine-induced acute itching. To further confirm the effect of osthole on acute histamine-dependent itch, we adopted a pretreatment by intradermal injection with osthole into the mouse neck before injection with histamine into the same site. As shown in Fig. 5, A and B, after intradermal injection of histamine $(100 \mu \mathrm{M})$ into the mouse nape, the number of spontaneous scratching bouts in histamine-treated mice significantly increased to $49.1 \pm 16.4(n=7, P<0.01)$ within 30 minutes compared to that of saline control at $3.4 \pm 3.9(n=5)$. With pretreatment of osthole $(300 \mathrm{nM})$, histamine-induced scratching bouts were significantly decreased to $9.0 \pm 10.0(n=6, P<0.01)$. To confirm whether histamine-induced itching is mediated by TRPV3 activation, we used the TRPV3-deficient mice and assessed the effect of

coaddition of $100 \mu \mathrm{M}$ osthole and $300 \mu \mathrm{M}$ AITC (3), and $20 \mu \mathrm{M}$ RR (4). (G) Summary for percentage inhibition of hTRPV3, human TRPV1, human TRPV4, and human TRPA1 channels by $100 \mu \mathrm{M}$ osthole. Data are presented as the means \pm S.D. Asterisks denote statistical significance as compared with hTRPV3 control $(n=5 ; * * * P<0.001$ by unpaired $t$ test $)$. 

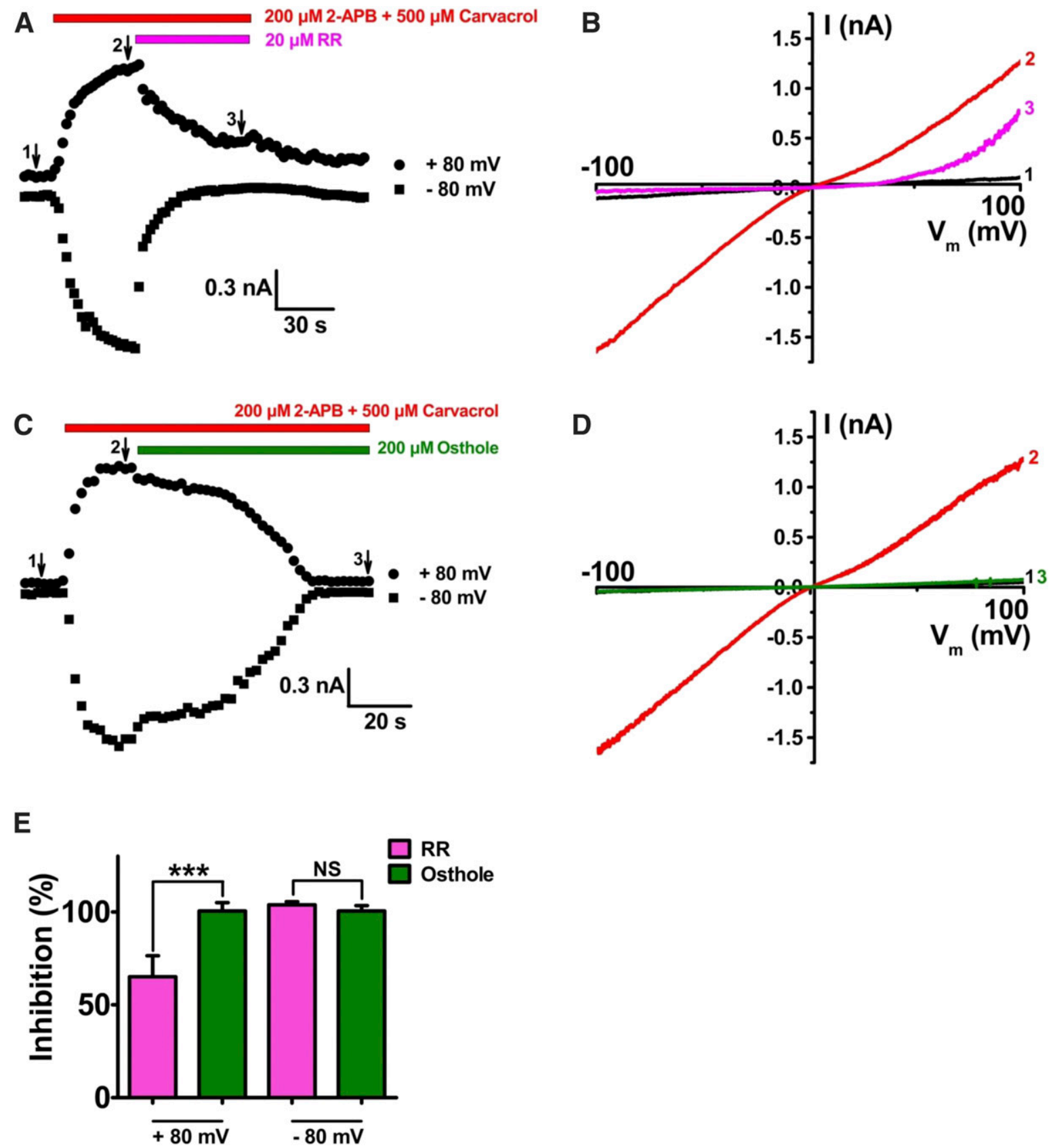

Fig. 3. Inhibition of endogenous TRPV3 current by osthole in mouse primary cultured keratinocytes. (A) TRPV3-like current was activated by TRPV3 agonist cocktail ( $200 \mu \mathrm{M} 2$-APB $+500 \mu \mathrm{M}$ carvacrol, red bar). The current was inhibited by coapplication of $20 \mu \mathrm{M}$ RR (pink bar). (B) Current-voltage curves of TRPV3 in response to voltage ramps from -100 to $+100 \mathrm{mV}$ under control conditions (1), $200 \mu \mathrm{M} 2-\mathrm{APB}+500 \mu \mathrm{M}$ carvacrol (2), and after coaddition of $20 \mu \mathrm{M}$ RR and TRPV3 agonist cocktail (3). (C) TRPV3-like current was activated by TRPV3 agonist cocktail (200 $\mu \mathrm{M} 2$-APB $+500 \mu \mathrm{M}$ carvacrol, red bar). The current was inhibited by coapplication of $200 \mu \mathrm{M}$ osthole (green bar). (D) Current-voltage curves of TRPV3 in response to voltage ramps from -100 to $+100 \mathrm{mV}$ under control conditions (1), $200 \mu \mathrm{M} 2$-APB $+500 \mu \mathrm{M}$ carvacrol (2), and after coaddition of $200 \mu \mathrm{M}$ osthole and TRPV3 agonist cocktail (3). (E) Summary for percentage inhibition of TRPV3-like current by $20 \mu \mathrm{M} R R$ and $200 \mu \mathrm{M}$ osthole at $\pm 80 \mathrm{mV}$. Data are presented as the means \pm S.D. $(n=5-6$; ***P $<0.001$ by one-way analysis of variance followed by Bonferroni's test). NS, not significant.

osthole on scratching behavior in TRPV $3^{-/}$mice. Silencing of TRPV3 resulted in a dramatic reduction of scratching induced by histamine as compared with wild-type TRPV $3^{+/+}$ mice. The number of scratching bouts induced by histamine in TRPV $3^{-1-}$ mice was significantly reduced to $10.3 \pm 9.4$ $(n=8, P<0.01)$ within 30 minutes as compared with $39.5 \pm$ $27.4(n=6)$ in wild-type TRPV3 ${ }^{+/+}$mice (Fig. 5, C and D). Further application of osthole $(300 \mathrm{nM})$ had no effect on reduction of scratching bouts in TRPV $3^{-1-}$ mice (Fig. 5, E and F). These results indicate that TRPV3 plays a pivotal role in mediating histamine-induced pruritus, and osthole suppresses histamine-induced scratching behavior primarily through inhibition of the TRPV3 channel.

\section{Discussion}

In this study, we identified natural coumarin osthole from the flowering plant $C$. monnieri as a novel and selective 

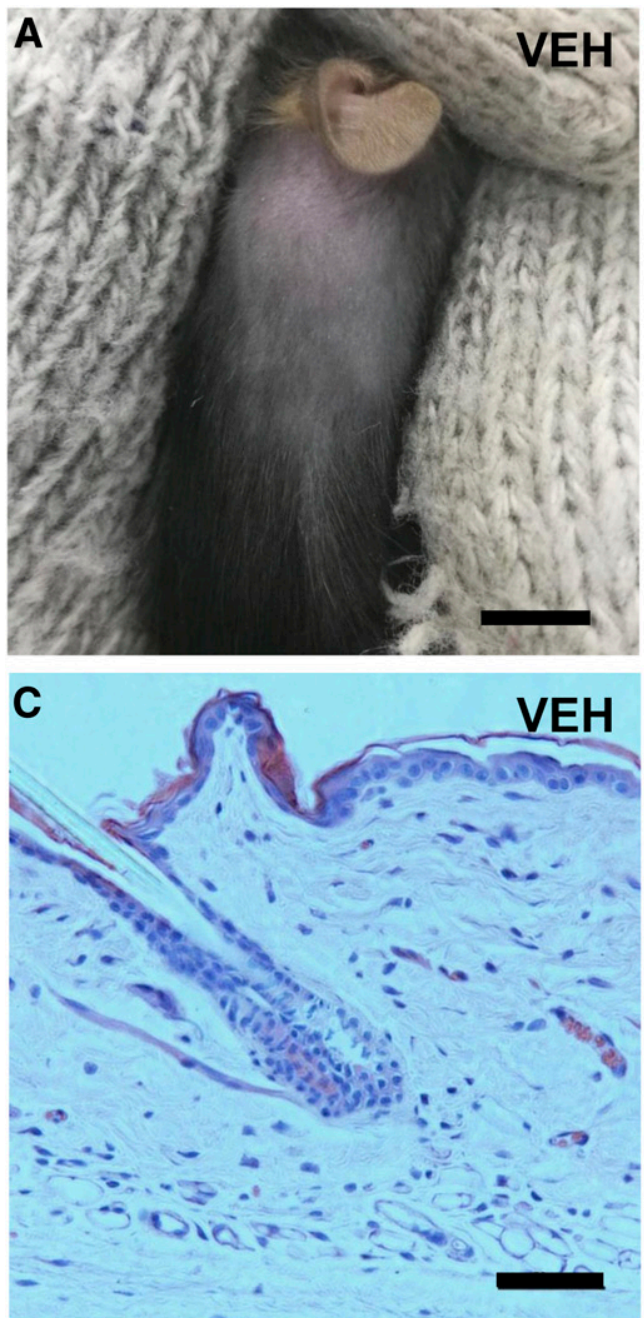

E

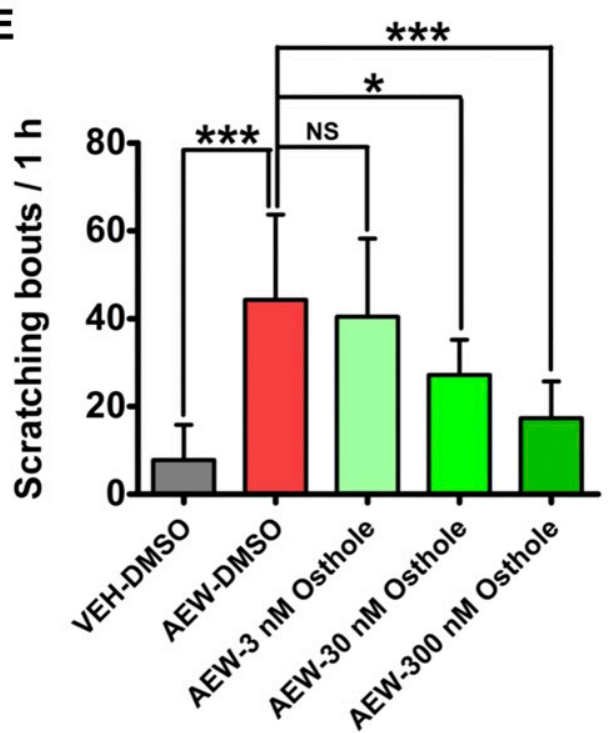

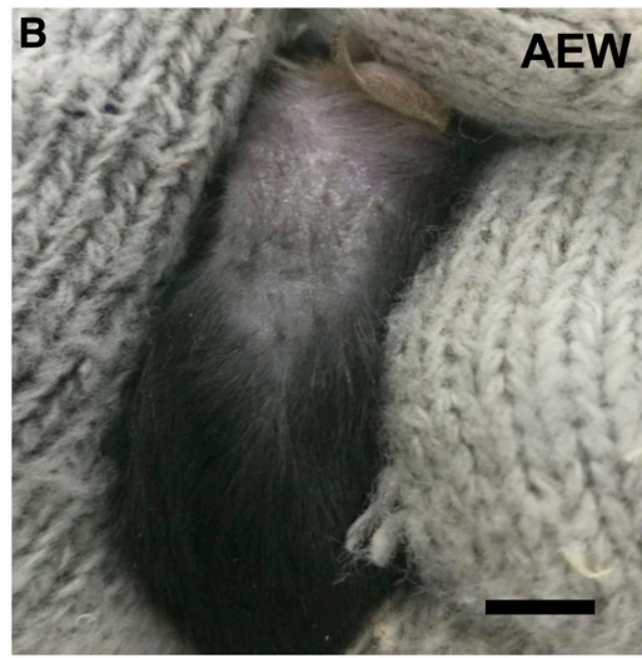

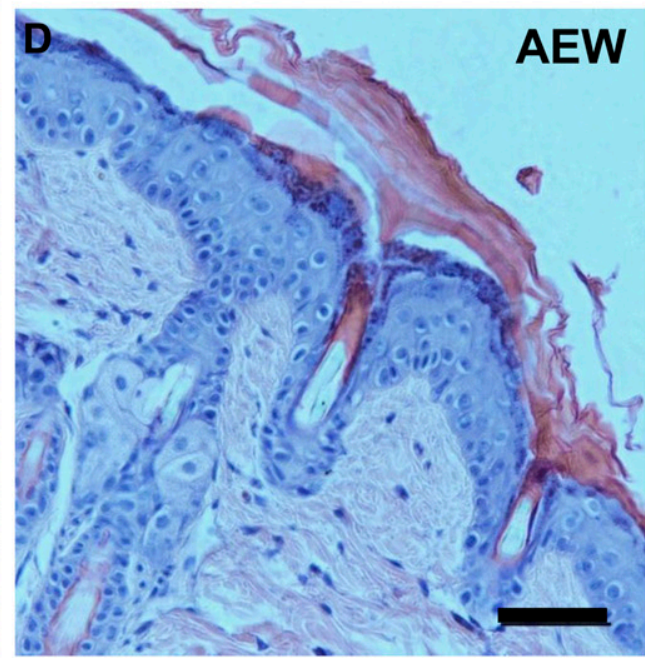

$\mathbf{F}$

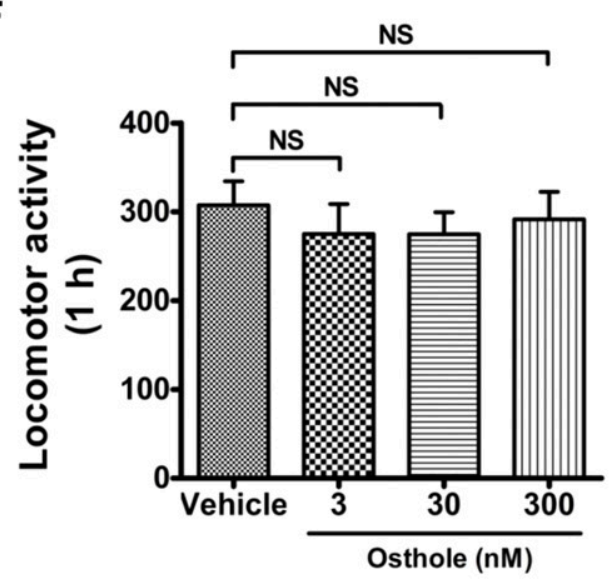

Fig. 4. The dose-dependent inhibition of AEW-induced scratching by osthole. (A and B) The illustrative comparison for development of dry skin in mice treated for 5 days with water VEH control and AEW. Scale bar, $1 \mathrm{~cm}$. (C and D) H\&E-stained skin tissue sections $(5 \mu \mathrm{m})$ from $\mathrm{VEH}$ and AEW. Scale bar, $50 \mu \mathrm{m}$. (E) The number of spontaneous scratching bouts with pretreatment of osthole was significantly attenuated in AEW-treated mice $(n=8-10 ; * P<0.05$, *** $P<$ 0.001 by one-way analysis of variance followed by Dunnett's test). (F) Locomotor activity (total travel distance, meters) had no significant alteration after intradermal injection with osthole $(3,30,300 \mathrm{nM}, 50 \mu \mathrm{l} / \mathrm{site})$ into the nape of the mouse neck $(n=6$; one-way analysis of variance followed by Dunnett's test). Data are presented as the means \pm S.D. NS, not significant. inhibitor of the TRPV3 channel. Whole-cell patch clamp recordings confirmed that osthole dose-dependently inhibits TRPV3 current activated in the presence of agonist 2-APB $(50 \mu \mathrm{M})$. Behavioral evaluations showed that osthole significantly attenuates mouse scratching behaviors induced by either AEW or histamine, but not in TRPV3-deficient mice. These findings demonstrate that TRPV3 plays a pivotal role in itch sensation, and specific suppression of TRPV3 function by natural osthole presents a therapeutic strategy for chronic itch or itch-related skin disorders. 

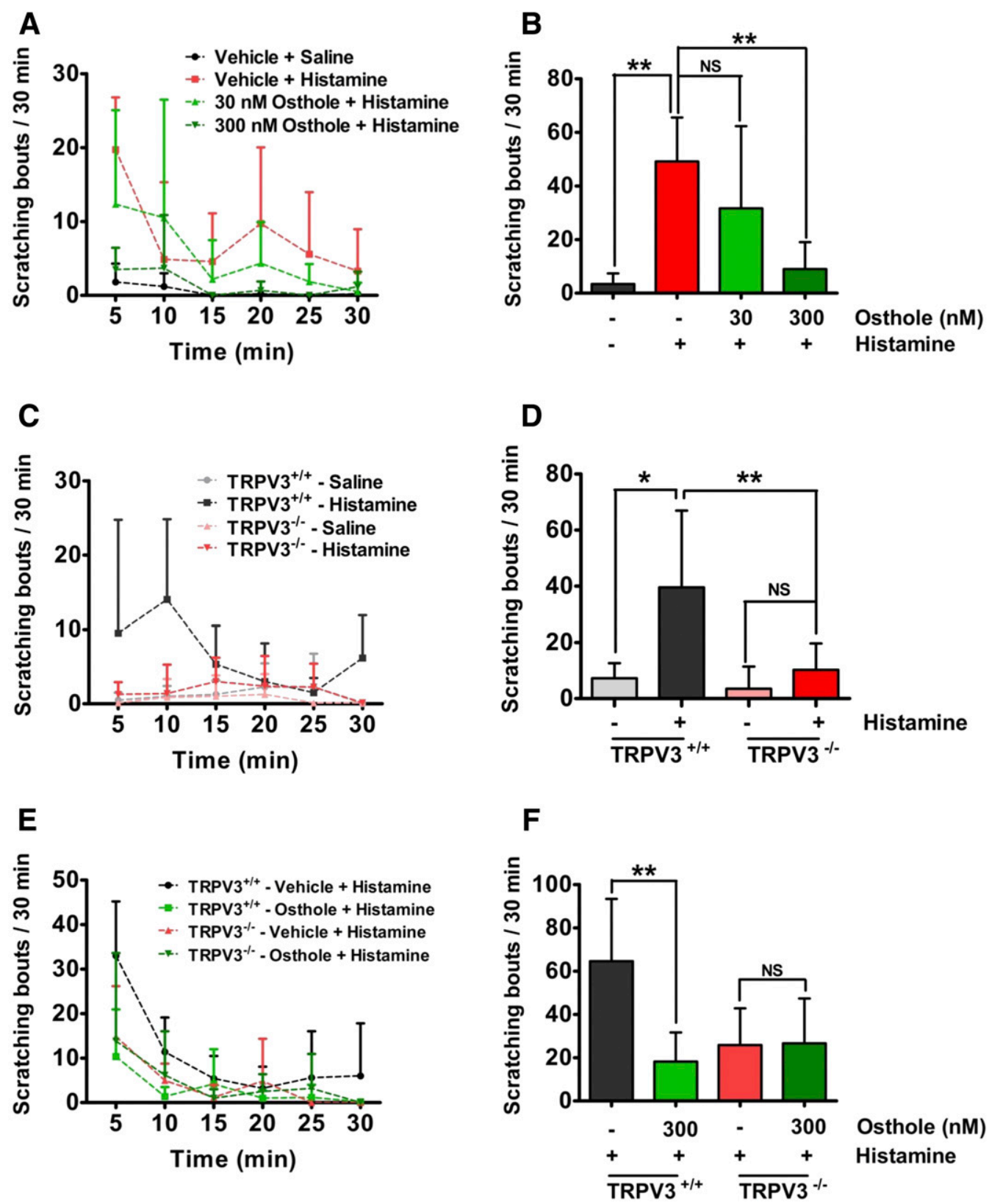

Fig. 5. Silencing or pharmacological inhibition of TRPV3 by osthole attenuates histamine-induced scratching. (A) The time course of neck-scratching activity was observed in C57BL/6 mice after intradermal injection histamine $(100 \mu \mathrm{M})$ in $50 \mu \mathrm{l}$ into mouse neck, with pretreatment of vehicle $(0.0003 \%$ DMSO) and different concentrations (30 and $300 \mathrm{nM}$ ) of osthole in $50 \mu$ linto the same site. Time for scratching bouts was plotted for each 5-minute bin over 30 minutes. (B) Quantification of scratching bouts during 30 minutes ( $n=5-7 ; * * P<0.01$ by one-way analysis of variance followed by Dunnett's test). (C) The time course of neck-scratching activity observed in TRPV $3^{++}$and TRPV $3^{-1-}$ mice after intradermal injection of histamine $(100 \mu \mathrm{M})$ in $50 \mu \mathrm{l}$ into the mouse neck. Time for scratching bouts was plotted for each 5-minute bin over 30 minutes. (D) Quantification of scratching bouts during 30 minutes $(n=4-8 ; * P<0.05, * * P<0.01$ by one-way analysis of variance followed by Bonferroni's test). (E) The time course of neck-scratching activity was observed in $\mathrm{TRPV}^{+/+}$and TRPV3 ${ }^{-/-}$mice after intradermal injection of histamine $(100 \mu \mathrm{M})$ in $50 \mu$ linto the mouse neck, with pretreatment of vehicle $(0.0003 \% \mathrm{DMSO})$ and $300 \mathrm{nM}$ osthole in $50 \mu \mathrm{l}$ into the same site. Time for scratching bouts was plotted for each 5-minute bin over 30 minutes. (F) Quantification of scratching bouts during 30 minutes $(n=5-6$; **P $<0.01$ by one-way analysis of variance followed by Bonferroni's test). Data are presented as the means \pm S.D. NS, not significant.

Although itch sensation has been studied at the spinal level (Sun and Chen, 2007; Sun et al., 2009; Mishra and Hoon, 2013) and at the central neural circuit through the spinoparabrachial pathway, which was recently identified to be activated during itch processing (Mu et al., 2017), the molecular targets critical for itch sensation at the peripheral skin level still remain largely unclear. Emerging evidence suggests that thermosensitive TRP channels (e.g., TRPV1, TRPV3, TRPV4,
TRPA1) not only act as "cellular sensors" on sensory neurons but also are functionally expressed in non-neuronal cells, such as the keratinocytes of the skin (Tóth et al., 2014). Both TRPV1 and TRPA 1 channels are expressed in nerve endings of cutaneous sensory afferent fibers involved in itch and pain sensation (Caterina and Pang, 2016). In acute histaminedependent itch, TRPV1 appears to function as a downstream target coupling ion channel and histamine receptor subtype 1. 
Acute histaminergic itch was significantly reduced by deletion of the TRPV1 gene or pretreatment with capsazepine, a TRPV1 blocker (Shim et al., 2007). In cultured sensory neurons from dorsal root ganglions, the TRPA1 channel has been shown to be a downstream target that binds to the chloroquine receptor MrgprA3, a Mas-related G proteincoupled receptor, and functions at the primary afferent C-fibers as an important component in acute histamineindependent itch signaling (Wilson et al., 2011). TRPA1deficient mice exhibit profound diminished responses to chloroquine-induced scratching (Wilson et al., 2011). The TRPV4 channel is not only expressed in primary afferents involved in the transduction of pain but is also highly expressed in skin epidermal keratinocytes (Shibasaki, 2016). However, whether TRPV4 plays an analogous role in itch is controversial. Akiyama et al. (2016) demonstrated that TRPV4 is a key mediator of histamine-independent itch associated with 5-hydroxytryptamine, which is not linked to histamine. In contrast, Chen et al. (2016) found that scratching behavior induced by histamine significantly reduced in TRPV4 knockout mice, suggesting TRPV4 as a pruriceptorTRP in skin keratinocytes in histamine-dependent itch. The role of TRPV4 in itch, or especially in histamine-induced itch, still requires further investigation and confirmation.

The identification of gain-of-function mutations of human TRPV3 from patients with Olmsted syndrome (OS) unveils the crucial role of the channel in chronic itch (Lin et al., 2012; Wang and Wang, 2017). TRPV3, a warm temperaturesensitive cation ion channel with an activation threshold of over $33^{\circ} \mathrm{C}$, is abundantly expressed in the keratinocytes of skin (Peier et al., 2002; Smith et al., 2002; Xu et al., 2002). It is a well known and commonly experienced that warmth facilitates itch sensation, suggesting that activation of TRPV3 is involved in itch processing. The TRPV3 Gly573Ser mutation caused spontaneous scratching behavior in C57BL-Nh mice (Yoshioka et al., 2009), revealing a role of TRPV3 in pruritic dermatoses (Steinhoff and Bíró, 2009). We also previously identified that human gain-of-function mutations of TRPV3 Gly573Ser and Trp692Gly cause the rare genetic disease OS characterized by severe itching and palmoplantar keratoderma (Lai-Cheong et al., 2012; Lin et al., 2012; Wilson et al., 2015). OS was first reported in an Italian American boy in 1927, and to date, about 80 clinical cases of OS have been reported and about 20 cases of OS were caused by TRPV3 gain-of-function mutations (Duchatelet and Hovnanian, 2015; Agarwala et al., 2016; Ni et al., 2016; Zhi et al., 2016). Severe itching in the skin lesions is a feature of OS, resulting in frequent scratching and insomnia (Lin et al., 2012). These observations indicate that TRPV3 plays a critical role in itch sensation and can serve as a potential target for itch therapy. Yet, there is a lack of specific inhibitors of TRPV3, which impedes the validation of TRPV3 as a therapeutic target in the skin that is essential for itch sensation and itch signaling. Therefore, it is necessary to identify specific inhibitors of TRPV3 for investigation of antipruritic effects.

The fruits of the $C$. monnieri annual plant, known as "She chuang zi" in China, have long been used as a traditional remedy for skin diseases. Many Chinese prescriptions for antipruritus contain the fruits of $C$. monnieri. For instance, "DahuangSan" can be used for treatment of female vaginal itching, and "Fufang Shechuangzi Xiji" can be used to remedy athlete's foot (Li et al., 2015). Because of the commonly use of
C. monnieri extracts in Chinese traditional medicines, several studies have investigated the active ingredients as well as their molecular mechanisms. The chloroform-soluble fraction from methanol extracts of $C$. monnieri markedly inhibits substance P-induced scratching (Basnet et al., 2001). The $70 \%$ ethanol extracts obtained from $C$. monnieri and osthole isolated from the $C$. monnieri extracts both had inhibitory effects on allergy (Matsuda et al., 2002). Many studies on C. monnieri have focused on antipruritic action of the extracts as well as the ingredients purified from the plant extracts in rodent animal models. Yet, the critical ingredients from the C. monnieri plant used for antipruritus and their mechanisms of action still remain elusive, although Yang et al. (2016) recently reported that osthole inhibits capsaicin-induced calcium influx and inward TRPV1 current in cultured small dorsal root ganglion neurons in which other TRPs channels are also expressed (Kittaka and Tominaga, 2017).

In this investigation, we identified osthole as a novel selective inhibitor of TRPV3 channels using a combination of calcium fluorescent assay and whole-cell patch clamp recordings. Although osthole also inhibits the TRPV1 channel, its efficacy on TRPV1 was significantly lower than TRPV3 (Fig. $2 \mathrm{G})$. To examine the antipruritic effect of osthole, we preferentially used the mouse model of chronic pruritus induced by AEW treatment (Yamamoto-Kasai et al., 2012). Our observations reveal that osthole as a selective TRPV3 antagonist attenuates dry skin itch induced by AEW treatment (Fig. 4E), indicating that pharmacological inhibition of TRPV3 in the skin may present an effective therapy for dry skin-induced itching. Our finding may also explain the molecular mechanism underlying any pruritic side effects of topical applications of natural extracts or cosmetic products from the fruit of C. monnieri that are involved in peripheral activation of thermosensitive TRPV3 channels in the skin.

Osthole not only inhibits chronic dry skin itch but also suppresses acute histamine-dependent itch (Fig. 5, A and B), which is consistent with the observation that osthole inhibits acute histamine-dependent itch by modulating TRPV1 activity (Yang et al., 2016). In TRPV1 knockout mice, neither neuronal calcium response nor behavioral scratching was completely eliminated, and some histamine-sensitive neurons failed to respond to capsaicin (Nicolson et al., 2002; Shim et al., 2007), suggesting other TRP channels are involved in acute histaminergic itch (Sun and Dong, 2016). Furthermore, infiltration of mast cells that release histamine was demonstrated by skin biopsies from keratotic lesions of OS individuals (Lin et al., 2012). These observations indicate that TRPV3 plays a critical role in acute histamine-dependent itch. Our data demonstrate that TRPV3-deficient mice display a significant reduction of scratching induced by histamine, and osthole had no effect on reduction of scratching bouts in TRPV $^{-1-}$ mice (Fig. 5, C-F). Therefore, we think that osthole suppresses histamine-induced scratching behavior primarily by inhibiting the TRPV3 channel.

In conclusion, our findings show that natural coumarin osthole from $C$. monnieri is a novel specific inhibitor of the TRPV3 channel that plays a critical role in itch sensation. Inhibition of peripheral thermosensitive TRPV3 in the skin by osthole may present antipruritic therapy or TRPV3-related skin diseases. Our findings may also prompt the potential of coumarins for remedies such as itch therapy or skin care products. 


\section{Acknowledgments}

We thank F. Hu, H. Li, Y. Song, X. L. Bian, and members of the Wang laboratory for providing assistance. X.-Y. S. is grateful for emotional and practical support from H. K. Yang. K. W. W. thanks J. M. Wang for consistent support.

\section{Authorship Contributions}

Participated in research design: X.-Y. Sun, G.-X. Wang, K.W. Wang.

Conducted experiments: X.-Y. Sun, L.-L. Sun, Gao.

Performed data analysis: X.-Y. Sun, Qi, Wei.

Wrote or contributed to the writing of the manuscript: X.-Y. Sun, K.W. Wang.

\section{References}

Agarwala MK, George R, Pramanik R, and McGrath JA (2016) Olmsted syndrome in an Indian male with a new de novo mutation in TRPV3. $\mathrm{Br} J$ Dermatol 174: 209-211.

Akiyama T, Ivanov M, Nagamine M, Davoodi A, Carstens MI, Ikoma A, Cevikbas F, Kempkes C, Buddenkotte J, Steinhoff M, et al. (2016) Involvement of TRPV4 in serotonin-evoked scratching. J Invest Dermatol 136:154-160.

Basnet P, Yasuda I, Kumagai N, Tohda C, Nojima H, Kuraishi Y, and Komatsu K (2001) Inhibition of itch-scratch response by fruits of Cnidium monnieri in mice. Biol Pharm Bull 24:1012-1015.

Cao X, Yang F, Zheng J, and Wang K (2012) Intracellular proton-mediated activation of TRPV3 channels accounts for the exfoliation effect of $\alpha$-hydroxyl acids on keratinocytes. J Biol Chem 287:25905-25916.

Caterina MJ and Pang Z (2016) TRP channels in skin biology and pathophysiology. Pharmaceuticals (Basel) 9:E77.

Chen Y, Fang Q, Wang Z, Zhang JY, MacLeod AS, Hall RP, and Liedtke WB (2016) Transient receptor potential vanilloid 4 ion channel functions as a pruriceptor in epidermal keratinocytes to evoke histaminergic itch. J Biol Chem 291: 10252-10262.

Cheng X, Jin J, Hu L, Shen D, Dong XP, Samie MA, Knoff J, Eisinger B, Liu ML, Huang SM, et al. (2010) TRP channel regulates EGFR signaling in hair morphogenesis and skin barrier formation. Cell 141:331-343.

Dien PH, Nhan NT, Le Thuy HT, and Quang DN (2012) Main constituents from the seeds of Vietnamese Cnidium monnieri and cytotoxic activity. Nat Prod Res 26 2107-2111.

Duchatelet S and Hovnanian A (2015) Olmsted syndrome: clinical, molecular and therapeutic aspects. Orphanet J Rare Dis 10:33.

Ikoma A, Steinhoff M, Ständer S, Yosipovitch G, and Schmelz M (2006) The neurobiology of itch. Nat Rev Neurosci 7:535-547.

Kittaka $\mathrm{H}$ and Tominaga M (2017) The molecular and cellular mechanisms of itch and the involvement of TRP channels in the peripheral sensory nervous system and skin. Allergol Int 66:22-30.

Lai-Cheong JE, Sethuraman G, Ramam M, Stone K, Simpson MA, and McGrath JA (2012) Recurrent heterozygous missense mutation, p.Gly573Ser, in the TRPV3 gene in an Indian boy with sporadic Olmsted syndrome. $\mathrm{Br} J$ Dermatol 167: 440-442.

Lei L, Cao X, Yang F, Shi DJ, Tang YQ, Zheng J, and Wang K (2013) A TRPV4 channel C-terminal folding recognition domain critical for trafficking and function. $J$ Biol Chem 288:10427-10439.

Li YM, Jia M, Li HQ, Zhang ND, Wen X, Rahman K, Zhang QY, and Qin LP (2015) Cnidium monnieri: a review of traditional uses, phytochemical and ethnopharmacological properties. Am J Chin Med 43:835-877.

Lin Z, Chen Q, Lee M, Cao X, Zhang J, Ma D, Chen L, Hu X, Wang H, Wang X, et al. (2012) Exome sequencing reveals mutations in TRPV3 as a cause of Olmsted syndrome. Am J Hum Genet 90:558-564.

Lucaciu OC and Connell GP (2013) Itch sensation through transient receptor potential channels: a systematic review and relevance to manual therapy. $J M a$ nipulative Physiol Ther 36:385-393.

Matsuda H, Tomohiro N, Ido Y, and Kubo M (2002) Anti-allergic effects of cnidii monnieri fructus (dried fruits of Cnidium monnieri) and its major component, osthol. Biol Pharm Bull 25:809-812.

Meng J and Steinhoff M (2016) Molecular mechanisms of pruritus. Curr Res Transl Med 64:203-206.

Mishra SK and Hoon MA (2013) The cells and circuitry for itch responses in mice. Science 340:968-971.

Miyamoto T, Nojima H, Shinkado T, Nakahashi T, and Kuraishi Y (2002) Itchassociated response induced by experimental dry skin in mice. Jpn $J$ Pharmacol 88:285-292.
Mollanazar NK, Smith PK, and Yosipovitch G (2016) Mediators of chronic pruritus in atopic dermatitis: getting the itch out? Clin Rev Allergy Immunol 51:263-292.

Moqrich A, Hwang SW, Earley TJ, Petrus MJ, Murray AN, Spencer KS, Andahazy M, Story GM, and Patapoutian A (2005) Impaired thermosensation in mice lacking TRPV3, a heat and camphor sensor in the skin. Science 307: 1468-1472.

Mu D, Deng J, Liu KF, Wu ZY, Shi YF, Guo WM, Mao QQ, Liu XJ, Li H, and Sun YG (2017) A central neural circuit for itch sensation. Science 357:695-699.

Ni C, Yan M, Zhang J, Cheng R, Liang J, Deng D, Wang Z, Li M, and Yao Z (2016) A novel mutation in TRPV3 gene causes atypical familial Olmsted syndrome. Sci Rep 6:21815.

Nicolson TA, Bevan S, and Richards CD (2002) Characterisation of the calcium responses to histamine in capsaicin-sensitive and capsaicin-insensitive sensory neurones. Neuroscience 110:329-338.

Peier AM, Reeve AJ, Andersson DA, Moqrich A, Earley TJ, Hergarden AC, Story GM Colley S, Hogenesch JB, McIntyre P, et al. (2002) A heat-sensitive TRP channel expressed in keratinocytes. Science 296:2046-2049.

Shibasaki K (2016) TRPV4 ion channel as important cell sensors. J Anesth 30: 1014-1019.

Shim WS, Tak MH, Lee MH, Kim M, Kim M, Koo JY, Lee CH, Kim M, and Oh U (2007) TRPV1 mediates histamine-induced itching via the activation of phospholipase A2 and 12-lipoxygenase. J Neurosci 27:2331-2337.

Smith GD, Gunthorpe MJ, Kelsell RE, Hayes PD, Reilly P, Facer P, Wright JE, Jerman JC, Walhin JP, Ooi L, et al. (2002) TRPV3 is a temperature-sensitive vanilloid receptor-like protein. Nature 418:186-190.

Steinhoff M and Bíró T (2009) A TR(I)P to pruritus research: role of TRPV3 in inflammation and itch. J Invest Dermatol 129:531-535.

Sun S and Dong X (2016) Trp channels and itch. Semin Immunopathol 38:293-307.

Sun YG and Chen ZF (2007) A gastrin-releasing peptide receptor mediates the itch sensation in the spinal cord. Nature 448:700-703.

Sun YG, Zhao ZQ, Meng XL, Yin J, Liu XY, and Chen ZF (2009) Cellular basis of itch sensation. Science 325:1531-1534.

Tóth BI, Oláh A, Szöllősi AG, and Bíró T (2014) TRP channels in the skin. $B r J$ Pharmacol 171:2568-2581.

Wang $\mathrm{G}$ and Wang $\mathrm{K}(2017)$ The $\mathrm{Ca}^{2+}$-permeable cation transient receptor potential TRPV3 channel: an emerging pivotal target for itch and skin diseases. Mol Pharmacol 92:193-200.

Wei NN, Lv HN, Wu Y, Yang SL, Sun XY, Lai R, Jiang Y, and Wang K (2016) Selective activation of nociceptor TRPV1 channel and reversal of inflammatory pain in mice by a novel coumarin derivative Muralatin L from Murraya alata. $J$ Biol Chem 291:640-651.

Wilson NJ, Cole C, Milstone LM, Kiszewski AE, Hansen CD, O’Toole EA, Schwartz ME, Irwin McLean WH, and Smith FJD (2015) Expanding the phenotypic spectrum of Olmsted syndrome. J Invest Dermatol 135:2879-2883.

Wilson SR, Gerhold KA, Bifolck-Fisher A, Liu Q, Patel KN, Dong X, and Bautista DM (2011) TRPA1 is required for histamine-independent, Mas-related G proteincoupled receptor-mediated itch. Nat Neurosci 14:595-602.

Wilson SR, Nelson AM, Batia L, Morita T, Estandian D, Owens DM, Lumpkin EA and Bautista DM (2013) The ion channel TRPA1 is required for chronic itch. $J$ Neurosci 33:9283-9294.

Xu H, Delling M, Jun JC, and Clapham DE (2006) Oregano, thyme and clove-derived flavors and skin sensitizers activate specific TRP channels. Nat Neurosci 9: 628-635.

Xu H, Ramsey IS, Kotecha SA, Moran MM, Chong JA, Lawson D, Ge P, Lilly J, SilosSantiago I, Xie Y, et al. (2002) TRPV3 is a calcium-permeable temperaturesensitive cation channel. Nature 418:181-186.

Yamamoto-Kasai E, Imura K, Yasui K, Shichijou M, Oshima I, Hirasawa T, Sakata T, and Yoshioka T (2012) TRPV3 as a therapeutic target for itch. J Invest Dermatol 132:2109-2112.

Yang NN, Shi H, Yu G, Wang CM, Zhu C, Yang Y, Yuan XL, Tang M, Wang ZL, Gegen T, et al. (2016) Osthole inhibits histamine-dependent itch via modulating TRPV1 activity. Sci Rep 6:25657.

Yoshioka T, Imura K, Asakawa M, Suzuki M, Oshima I, Hirasawa T, Sakata T, Horikawa T, and Arimura A (2009) Impact of the Gly573Ser substitution in TRPV3 on the development of allergic and pruritic dermatitis in mice. $J$ Invest Dermatol 129:714-722.

Zhang X (2015) Targeting TRP ion channels for itch relief. Naunyn Schmiedebergs Arch Pharmacol 388:389-399.

Zhi YP, Liu J, Han JW, Huang YP, Gao ZQ, Yang Y, and Wu RN (2016) Two familial cases of Olmsted-like syndrome with a G573V mutation of the TRPV3 gene. Clin Exp Dermatol 41:510-513.

Address correspondence to: KeWei Wang, Department of Pharmacology, Qingdao University School of Pharmacy, 38 Dengzhou Road, Qingdao 266021, Shandong Province, China. E-mail: wangkw@qdu.edu.cn 